\title{
ABORDAJE DE LA CULTURA DESDE LA INVESTIGACIÓN EN CONTABILIDAD: POSIBILIDADES DE RE-SIGNIFICACIÓN ${ }^{\star}$
}

\author{
JORGE ANDRÉS SALGADO CASTILLO** \\ UNIVERSIDAD MILITAR NUEVA GRANADA
}

Recibido/ Received/ Recebido: 13/08/14 - Aceptado/ Accepted / Aprovado: 23/10/15

\begin{abstract}
Resumen
El concepto de cultura se ha trabajado en contabilidad principalmente para evaluar las diferencias en los sistemas contables y en el control de gestión entre países, lo anterior tiene por objeto caracterizar y sobre todo comparar los elementos principales que impactan a la contabilidad y el control en las culturas nacionales. El trabajo de Hofstede $(1980,1984)$ es el referente principal de la investigación contable en el ámbito de la cultura, sin embargo no escapa de cuestionamientos por no tomar en cuenta las construcciones de disciplinas que estudian el fenómeno cultural, tales como la antropología cultural, la historia, la sociología, entre otras. El presente trabajo expone los marcos y referentes de la antropología cultural, como elemento importante del análisis de la cultura, posteriormente, se esbozan los referentes utilizados por la contabilidad para el estudio de la cultura, la aplicación en sus estudios y las críticas surgidas.

Palabras clave: Contabilidad; Control; Cultura; Dimensiones culturales; Enfoques; Investigación en contabilidad.
\end{abstract}

\section{CULTURE APPROACH FROM ACCOUNTING RESEARCH: POSSIBILITIES OF RE - SIGNIFICANCE}

\begin{abstract}
The concept of culture has worked in accounting primarily to evaluate differences in accounting systems and management control between countries, the above is intended to characterize and compare all the main elements that impact accounting and control in the national cultures. Hofstede's work $(1980,1984)$ is the main reference for accounting research in the field of culture, however it does not escape questioning by not taking into account the construction of disciplines that study the cultural phenomenon, such as cultural anthropology, history, sociology, among others. This paper outlines the frames and benchmarks of cultural anthropology as an important element of the culture
\end{abstract}

\footnotetext{
El presente texto es producto de los proyectos de investigación: "Clasificación internacional de los sistemas de contabilidad de gestión”, Vigencia 2011 (Código ECO-834), y “Cultura y sistemas de contabilidad de gestión: estudio de caso en el GSED” Vigencia 2013 (Código ECO-1223). Ambos proyectos fueron financiados por la Vicerrectoría de Investigaciones de la Universidad Militar Nueva Granada.

* Contador Público de la Universidad del Valle (Colombia), Magíster en Gestión de Organizaciones de la Universidad Militar Nueva Granada (Colombia). Profesor del programa de Contaduría Pública y miembro del Grupo de Estudios Contemporáneos en Contabilidad, Gestión y Organizaciones de la Universidad Militar Nueva Granada. Dirección postal: Cra. 11 No 101-80. Bloque C, Piso 1. Teléfono: 6500000 Ext. 1306. Correo electrónico: jorge.salgado@unimilitar.edu.co.
} 
analysis, subsequently the references used by accounting for the study of culture are outlined, the application in its studies and the emerging criticism.

Keywords: Accounting; Control; Culture; Cultural dimensions; Approaches; Research in accounting.

\title{
ABORDAGEM DA CULTURA DESDE A PESQUISA EM CONTABILIDADE: POSSIBILIDADES DE RE-SIGNIFICAÇÃO
}

\begin{abstract}
Resumo
O conceito de cultura trabalhou-se em contabilidade principalmente para avaliar as diferenças nos sistemas contábeis e no controle de gerenciamento entre países, com o fim de caracterizar e sobretudo comparar os elementos principais que impactam sobre a contabilidade e sobre o controle nas culturas nacionais. O trabalho de Hofstede $(1980,1984)$ é o referente principal da pesquisa contábil no âmbito da cultura. No entanto, não escapa de questionamentos por não considerar as construções de disciplinas que estudam o fenômeno cultural, tais como a antropologia cultural, a história, a sociologia, entre outras. O presente trabalho expõe os contextos e referentes da antropologia cultural, como elemento importante da análise da cultura. Posteriormente, se esboçam os referentes utilizados pela contabilidade para o estudo da cultura, a aplicação em seus estudos e as críticas surgidas. Palavras chave: Contabilidade; Controle; Cultura; Dimensões culturais; Enfoques; Pesquisa em contabilidade.
\end{abstract}

Salgado, J. (2016). Abordaje de la cultura desde la investigación en contabilidad: posibilidades de re-significación. En: Revista de la Facultad de Ciencias Económicas de la Universidad Militar Nueva Granada. rev.fac.cienc.econ, XXIV No1, DOI: http://dx.doi.org/10.18359/rfce.1624.

JEL: $M 40, M 41$

\section{Introducción}

El estudio de los sistemas contables y de los sistemas de control de gestión a nivel internacional, muestra como uno de los aspectos más importantes para evaluar y comparar las diferencias entre países, los aspectos culturales. La construcción y la réplica de variables y dimensiones que puedan explicar las diferencias entre culturas desde el ámbito de la contabilidad y el control, han sido la respuesta desde la disciplina contable ante el estudio de la cultura y las diferencias culturales, sin embargo, los presupuestos y las corrientes sobre las cuales se construyen las dimensiones culturales se cuestionan por algunos investigadores Schein (2010), Bhimani (1999), Harrison \& Mckinnon (1999), Chenhall (2003), entre otros. Lo anterior abre un debate que el presente artículo intenta abordar para alimentar la futura investigación contable que se enfoca en la cultura.
Las disciplinas que originaron el debate acerca de la cultura mantienen hasta hoy serias diferencias en los enfoques y concepciones fundamentales del concepto cultural, sin embargo, es claro que la cultura es un fenómeno complejo y está relacionada con la vida en sociedad y se puede tomar como una dimensión analítica de la misma que se materializa en contextos y tiempos determinados que son determinantes de la misma cultura. En contabilidad, las investigaciones que han tenido dentro de sus preocupaciones el fenómeno cultural, han utilizado el marco referencial de Hofstede (1980) y su formulación de dimensiones culturales. Así mismo, los temas trabajados desde el ámbito de la Contabilidad y el concepto cultural han estudiado el campo de la clasificación de los sistemas contables y las diferencias internacionales en los mismos, la cultura como factor a tener en cuenta en el análisis de los cambios en la regulación de los reportes financieros corporativos, el impacto de la cultura sobre la divulgación de la información financiera y la 
importancia de las variables culturales en el campo de los sistemas de control de gestión.

El presente trabajo tiene como objetivo aportar elementos que ayuden a re-significar el concepto de cultura que se ha estudiado en contabilidad. Para lo anterior, se mostrará la visión de la antropología cultural, en donde se tiene en cuenta la precisión conceptual de sus definiciones y los enfoques y tendencias de trabajo en esta área del saber, aquello es importante, ya que se constituye como disciplina base del estudio de la cultura. La mirada de la cultura desde las organizaciones que se muestra cercana a la Contabilidad es la que brinda el estudio de las organizaciones y la Administración Empresarial, se muestran entonces algunos elementos que son importantes desde el enfoque de la denominada "cultura organizacional". El marco de trabajo utilizado en los estudios de cultura y contabilidad es el aportado por Hofstede (1980), este importante marco se explica a profundidad desde sus concepciones conceptuales, para comprender la posterior utilización de las denominadas dimensiones culturales a los estudios en contabilidad. Posteriormente, se muestran los principales trabajos en la línea de investigación en contabilidad y cultura, para tener mayores elementos con respecto a cómo se ha estudiado el fenómeno desde el campo contable. Finalmente, se aportan discusiones que pueden ser objeto de iniciación de futuros trabajos.

\section{Visión amplia y diversa de la cultura: el aporte de la antropología cultural}

La antropología busca el modo de alcanzar el conocimiento sobre la humanidad, por tanto, tiene en cuenta el acercamiento a pueblos y a tierras, próximas y lejanas, así como de épocas remotas y actuales. La antropología cultural, estudia las tradiciones aprendidas de pensamiento y conducta denominadas culturas, indagando su surgimiento, intentando explicar los cambios de las mismas, y sus criterios de diferenciación. El estudio de las culturas seguido por la antropología cultural se consagra a la descripción y comparación sistemática de culturas, proporcionando elementos que sirven para formular hipótesis y reforzar teorías sobre las causas de los estilos de vida (Harris, 2009). Diversas disciplinas del conocimiento se han apoyado en la antropología cultural con mayor o menor éxito en la búsqueda de estudiar sus objetos particulares, sin embargo, el apoyo que brindan otras disciplinas del conocimiento debe ser examinado detenidamente, para evaluar el real aporte que han hecho y el uso que le han dado los investigadores.

Los diferentes enfoques, escuelas de pensamiento y tendencias de investigación en antropología cultural, plantean así mismo, diversidad de conceptos que enriquecen el mismo concepto de cultura y su estudio posterior. Una visión clásica de la cultura la concibe como un todo que incluye las creencias, el conocimiento, el arte, el derecho, las costumbres y cualquier hábito y capacidad adquirido por el hombre como miembro de la sociedad, este enfoque denominado "evolucionista" fue el punto de partida obligado del extenso debate sobre la cultura entablado hasta mediados del siglo XX. El anterior enfoque estableció rigidez en sus esquemas, ante lo cual otros enfoques (históricos) afirmaron la pluralidad histórica de las culturas, pasando entonces a una objetividad relativa basada en las características de cada cultura.

Posterior a las fases evolucionista e histórica, el concepto de cultura pasa por tres fases (Giménez, 2007). En primer lugar, la denominada "fase concreta" define a la cultura como un conjunto de costumbres, de manera específica como las formas y modos de vida que definen las características de un pueblo y lo identifican. En segundo lugar, en la "fase abstracta" la cultura se define en términos de modelos, parámetros o esquemas de comportamiento, este esquema pone atención a los sistemas de valores y a las estructuras de tipo normativo que regulan e influyen en los comportamientos de los individuos que pertenecen a un colectivo social. Finalmente, "la fase simbólica" define la cultura como estructuras de significados establecidos socialmente, estas estructuras o redes de significados pueden ser interpretadas como un texto que debe ser interpretado por el antropólogo cultural.

Geertz (2003) define la cultura, como un esquema de significaciones representadas en símbolos, 
históricamente trasmitido, es decir, un sistema de concepciones heredadas, expresadas en formas simbólicas por medios, con los cuales el hombre comunica, perpetúa y desarrolla su conocimiento y sus actitudes frente a la vida. El proceso de enculturación ${ }^{11}$, depende de la capacidad humana de utilizar símbolos. Por intermedio del aprendizaje cultural, las personas crean, recuerdan y manejan ideas, bajo el control y aplicación de sistemas específicos de significado simbólico, de forma consciente o inconsciente (Kottak, 2006). Los sistemas de símbolos creados por el hombre, compartidos, convencionales, y, por cierto, aprehendidos, suministran a los seres humanos un marco significativo dentro del cual pueden orientarse en sus relaciones recíprocas, en su relación con el mundo que los rodea y en su relación consigo mismos (Geertz, 2003; Salgado \& Caballero, 2013a).

La cultura, también puede comprenderse como una serie de mecanismos de control, semejantes a los planos para el arquitecto, las recetas para el cocinero, los programas para la computación. Son reglas e instrucciones que pueden impactar en el comportamiento de los integrantes de una colectividad (Kottak, 2006). Estos programas son absorbidos por el hombre en el proceso de enculturación. La concepción de la cultura desde el punto de vista de "mecanismos de control" comienza con el supuesto de que el pensamiento humano es fundamentalmente social y público, de que su lugar natural es el patio de la casa, la plaza de mercado y la plaza de la ciudad (Geertz, 2003; Salgado \& Caballero, 2013a).

Las inquietudes que se pueden catalogar como de carácter antropológico son tan antiguas como nuestra especie, los miembros de diferentes grupos humanos, en diferentes estancias geográficas y temporales, han tenido siempre curiosidad por las costumbres y tradiciones de los extraños (Harris, 2009). Las diferentes teorías sobre la cultura, han contribuido en las discusiones sobre esas mismas costumbres y tradiciones, además han servido de marco referencial de diferentes disciplinas además

1 Proceso por el cual una persona adquiere los usos, creencias, tradiciones, etc., de la sociedad en que vive (RAE, 2013). de la antropología, en la dirección de generar horizontes de comprensión de ciertos comportamientos humanos. Los enfoques nos permiten observar la evolución y la pluralidad de ideas y pensamientos alrededor de la cultura, estudiarlos permitirá establecer mayor rigor en las construcciones conceptuales que pudieran hacerse desde otras disciplinas.

El análisis de las culturas es central para los estudios aplicados que pretenden comprender los aspectos particulares que se estudian en sus objetos particulares, una manera de analizar los hechos culturales es la planteada por Giménez (2000) en la cual esboza tres dimensiones de análisis: i) la cultura como comunicación: están incluidos los conjuntos de sistemas, signos, señales, el lenguaje, el hábitat, entre otros, considerándolos como sistemas semióticos, ii) La cultura como stock de conocimiento: se incluyen las creencias, el conocimiento práctico, la ciencia, el sentido común, la intuición, la contemplación, entre otros, iii) La cultura como visión del mundo: se incluyen todas las interpretaciones del mundo, tales como las religiones, las filosofías y las ideologías. Las tres dimensiones anteriores pueden reflejarse en la cultura específica de una colectividad, se puede hablar de una síntesis que delimita de manera analítica la capacidad de creación e innovación de una colectividad, su facultad de adaptarse y la voluntad de intervenir sobre sí misma y su entorno.

La cultura contribuye a la existencia de una colectividad en la medida en que constituye su memoria, contribuye a cohesionar sus actores y permite legitimar sus acciones, la cultura es a la vez determinada y determinante.

\section{Cultura: una mirada desde las orga- nizaciones}

Desde una perspectiva asociada a las organizaciones empresariales, Schein (2010) realiza una aproximación a la cultura organizacional a partir de una perspectiva funcionalista. Se establece que los grupos sociales (incluidas las organizaciones) enfrentan dos problemas comunes: el primero, sobrevivir y adaptarse a los cambios del entorno, y segundo, la integración de los procesos internos para asegurar la 
Tabla 1. Enfoques y corrientes de la antropología cultural

\begin{tabular}{|c|c|}
\hline Enfoques & Ideas centrales \\
\hline La ilustración & $\begin{array}{l}\text { Primeros intentos sistemáticos de ofrecer teorías de las diferencias culturales. } \\
\text { El enfoque estaba dado por la evolución del progreso de un estado "natural" a un estado de civilización "ilustrada". } \\
\text { Las diferencias culturales son resultado del progreso intelectual y moral de los pueblos. }\end{array}$ \\
\hline $\begin{array}{l}\text { Evolucionismo del } \\
\text { siglo XIX }\end{array}$ & $\begin{array}{l}\text { Las teorías de la cultura estuvieron dominadas durante el siglo XIX por el concepto de evolución cultural. } \\
\text { Las culturas se consideraban generalmente en movimiento a través de diversas etapas de desarrollo (salvajismo, barbarie, civiliza- } \\
\text { ción), siendo la civilización la cúspide, algo similar a los estilos de vida euroamerizanos modernos. }\end{array}$ \\
\hline Darwinismo social & $\begin{array}{l}\text { Plantea la evolución de la cultura en conjunción con la evolución de los tipos y razas biológicas humanas (se seguía considerando } \\
\text { las culturas europeas y americanas como las de mayor progreso cultural). } \\
\text { Raza blanca como el pináculo del progreso biológico. } \\
\text { Las ideas de selección natural y supervivencia del más apto se tomaron para justificar varios hechos culturales. }\end{array}$ \\
\hline Evolucionismo marxista & $\begin{array}{l}\text { Observa las culturas pasando por las etapas de comunismo primitivo, sociedad esclavista, feudalismo, capitalismo y comunismo. } \\
\text { Se recalca el papel de la lucha entre clases sociales por el control de los medios de producción, en el logro del progreso y la } \\
\text { evolución cultural. }\end{array}$ \\
\hline Particularismo histórico & $\begin{array}{l}\text { Reacciona contra los esquemas y doctrinas evolucionistas. } \\
\text { Para comprender una cultura en particular, debe reconstruirse la trayectoria única que ha seguido (cada cultura tiene su historia). } \\
\text { Introduce la noción de "relativismo cultural", que mantiene que no existen formas inferiores o superiores de cultura. } \\
\text { Se critica las clasificaciones de "salvajismo", "barbarie" y "civilización", ya que expresa el etnocentrismo y el pensamiento de que } \\
\text { hay formas de vivir más normales o mejores que otras. }\end{array}$ \\
\hline Difusionismo & $\begin{array}{l}\text { La fuente de las diferencias y similitudes de las culturas es la tendencia de los humanos a copiarse, a imitarse entre si. } \\
\text { Los difusionistas consideran las culturas como un mosaico de elementos derivados de una serie fortuita de préstamos entre pueblos } \\
\text { cercanos y distantes. }\end{array}$ \\
\hline $\begin{array}{l}\text { Funcionalismo } \\
\text { y funcionalismo } \\
\text { estructural }\end{array}$ & $\begin{array}{l}\text { El funcionalismo pretende descubrir las funciones recurrentes de las costumbres e instituciones, antes que explicar los orígenes de } \\
\text { las similitudes y diferencias culturales. Una vez que se haya entendido la función de una institución, se puede entender todo lo que se } \\
\text { puede comprender sobre sus orígenes. } \\
\text { El funcionalismo estructural enfatiza en la contribución del bienestar biológico y psicológico de los individuos al mantenimiento del } \\
\text { sistema social. La función de mantener el sistema tiene prioridad sobre todas las demás. }\end{array}$ \\
\hline Cultura y personalidad & $\begin{array}{l}\text { Relacionan las creencias y prácticas culturales con la personalidad individual, y la personalidad individual con las creencias y } \\
\text { prácticas culturales. }\end{array}$ \\
\hline Nuevo evolucionismo & $\begin{array}{l}\text { Lo nuevo de este evolucionismo es que no se postula un conjunto sencillo o unilineal de etapas para todas las culturas, sino que } \\
\text { existen muchas o "multilineales" trayectorias de desarrollo, volviéndose una red compleja, y dependiente de las condiciones am- } \\
\text { bientales, tecnológicas, entre otras. } \\
\text { Se sentaron las bases de la ecología cultural, que recalcaba el papel de la interacción de condiciones naturales (tierra, lluvias, } \\
\text { temperatura) con factores culturales como la tecnología y la economía como causas de las diferencias y similitudes culturales. }\end{array}$ \\
\hline Materialismo dialéctico & $\begin{array}{l}\text { Para comprender las causas de las diferencias y similitudes socio culturales, no sólo se deben estudiar las contradicciones inter- } \\
\text { nas de los sistemas socio culturales, sino que se debe tomar parte en las resoluciones dialécticas que conducen al progreso del } \\
\text { comunismo. }\end{array}$ \\
\hline Materialismo cultural & $\begin{array}{l}\text { Las causas más probables de diferencia en los aspectos mentales o espirituales de la vida humana, son las variaciones en las } \\
\text { limitaciones materiales que afectan la forma en que las personas se enfrentan con los problemas de satisfacer las necesidades } \\
\text { básicas en un hábitat particular. }\end{array}$ \\
\hline
\end{tabular}

Fuente: Harris (2009).

capacidad de seguir sobreviviendo y adaptarse. La cultura está integrada al concepto de grupo social ya que, en palabras de Schein (2010), sin un grupo no puede existir una cultura y para la formación del grupo es necesario que haya ciertos supuestos compartidos, es decir, un grado mínimo de cultura. Sin estos supuestos compartidos no sería posible hablar de un grupo social, sino que solo se podría hablar de un conjunto de personas, de experiencias y actividades compartidas.

El análisis de una cultura determinada exige la delimitación del grupo social que se pretende estudiar, además del enfoque y definición que se adopte del 
concepto de cultura. Schein (2010) propone varias categorías de acuerdo a los grupos que se pretenda abarcar. En primer lugar, las denominadas "culturas macro", en esta categoría están las Naciones, los grupos étnicos y religiosos y los oficios que existen globalmente. Paso seguido están las denominadas "culturas de la organización" o "culturas organizacionales" que incluyen las organizaciones públicas y privadas, además de las organizaciones sin ánimo de lucro. Al interior de las organizaciones se encuentran las "subculturas" que incluyen los grupos de oficios al interior de las organizaciones. Finalmente, se encuentran las "culturas micro" que son aquellos microsistemas dentro o fuera de las organizaciones.

Mucho de lo que ocurre en una organización que ha existido por algún tiempo puede ser entendido como un conjunto de interacciones de subculturas operando en el contexto mayor de la cultura organizacional. Estas subculturas comparten mucho de los supuestos de la organización en conjunto, pero también mantienen supuestos que van más allá de los de la organización que usualmente reflejan sus tareas operativas, la ocupación de sus miembros o sus experiencias únicas. Es importante anotar que muchas de esas subculturas tienen su origen en ocupaciones mayores como la medicina o la ingeniería, que tienen supuestos de una base mayor, incluso internacional.

El concepto de "cultura organizacional" puede concebirse como un patrón socialmente construido y validado de supuestos básicos compartidos, que han sido desarrollados por un grupo específico de personas (miembros de la organización), y que sirve como medio de aprendizaje para hacer frente a los problemas de adaptación externa e integración interna (Batool, 2011; Busco \& Scapens, 2011; Agbejoule, 2011). Tales supuestos que se dan por sentado representan el almacén del conocimiento organizacional, que se "enseña" a los recién llegados como la manera adecuada de actuar, pensar y sentir en relación con situaciones específicas que surgen en el desarrollo organizacional (Salgado \& Caballero, 2013a). La cultura organizacional podría concebirse también como un "conjunto de postulados" compartidos por los miembros, dirigentes y empleados. Sería un "cemento" que "mantiene" la organización como un todo", que le da un "sentido" y un "sentido de identidad" para sus miembros. Sería también un "sistema de representaciones y de valores compartidos" que harían que "cada uno en la empresa, adhiera a una visión común de lo que es la empresa", un "compromiso" del personal hacia una entidad unificadora, la empresa, concebida como un "cemento social", un "conjunto de creencias, de valores y de normas" que constituye "modelos de comportamiento", un conjunto de "símbolos", de "significaciones" y de "objetivos" compartidos. (Aktouf, 2002, p. 65). Se concluye que independiente de la escuela, la cultura organizacional pretende la comunión de todos, patrones y obreros, dirigentes y dirigidos, en un mismo y entusiasta movimiento de apoyo de la empresa y de sus objetivos (Aktouf, 2002).

Aktouf (2002) analiza brevemente las corrientes del management que se ocupan de la cultura organizacional, destacando los pasos iniciales del comparative management hacia finales de los años cincuenta y durante los años sesenta, dicho movimiento se centró principalmente en los problemas alrededor de la internacionalización de las actividades industriales y la confrontación de las diferentes culturas en el mundo. Este enfoque comparativo, intentó indagar la gestión al modo chino, soviético, europeo y de analizar lo que los acercaba y lo que las alejaba del management norteamericano, posteriormente el enfoque se dirigió hacia Japón sobre todo a finales de los años setenta y comienzos de los años ochenta, esta mirada hacia el Japón se dio por la revolución japonesa del management que obligo a EUA y a otros países a mirar prácticas de gestión diferentes que lograban resultados envidiables. En aquella mirada se empezó a considerar a la empresa como una entidad social y como una entidad susceptible de producir sus propias reglas, costumbres, hábitos, visiones, lenguajes, es decir, que emerge una cultura propia de la empresa que la diferencia de otras, aún si unas y otras pertenecen a una cultura global común (Aktouf, 2002).

En las organizaciones japonesas que tienen un enfoque hacia el compromiso y el desempeño, la organización puede trabajar como una comunidad o como una familia con una misión de "calidad" y "servicio" (Herath, 2007). Ouchi (1979) plantea un marco relacionado con la cultura, este marco incluye la noción 
de control del clan, que es el control social ejercido sobre los individuos que hacen parte de un grupo y se identifican con ese grupo y sus valores (Salgado, 2015). Este estudio resalta la importancia de considerar a la cultura cuando se piensa en el control organizacional, también pone de relieve la dificultad de lograr un resultado deseado por imposición (Berry, Broadbent \& Otley, 2005). No se puede suponer que la cultura puede ser impuesta como un medio de control (Merchant \& Otley, 2007; Salgado, 2015).

\section{Estudios comparativos de culturas na- cionales: referente principal de la in- vestigación en contabilidad y cultura}

La cultura, afirma Hofstede (2011), es un fenómeno colectivo, esto podría tomarse como referencia al estudio de las culturales nacionales; dentro de cada colectividad hay una variedad de individuos y subculturas que influyen sobre las culturas nacionales y son influenciadas por ellas. Por lo general el término cultura se utiliza para tribus o grupos étnicos (en antropología), para las naciones (en ciencia política, sociología y gestión), y para las organizaciones (en sociología y gestión). Un campo relativamente inexplorado es la cultura de las ocupaciones (por ejemplo, de los ingenieros frente a los contables, o de académicos de distintas disciplinas). El término también se puede aplicar a los géneros, a las generaciones, o a las clases sociales.

Las culturas nacionales que son de corte social se trasladan desde la niñez y la juventud mediante la familia, la educación (en diversas instancias), las relaciones sociales, entre otras variables, Hofstede (2011) afirma que esas estructuras culturales tienen raíces más profundas en la mente humana que las culturas laborales o profesionales (adquiridas en ciertos niveles de formación) y las culturas organizacionales adquiridas en el trabajo. Las culturas sociales residen (a menudo de manera inconsciente) en los valores, en el sentido de la tendencia a preferir ciertos estados de cosas sobre otros, las culturas organizacionales residen más bien (de manera visible y consciente) en las prácticas, es decir, en la forma de percibir los que sucede en su entorno organizacional (Hofstede, 2011).
Los trabajos de Hofstede ${ }^{2}(1980,1984)$ son los que más se han utilizado en la comparación de culturas nacionales y sistemas contables a nivel mundial, los trabajos toman como base las denominadas dimensiones culturales ${ }^{3}$. En la actualidad se han formulado seis dimensiones de cultura nacional: i) distancia al poder, ii) Individualismo, iii) Aversión a la incertidumbre, iv) Masculinidad, v) Orientación al largo plazo, y vi) Indulgencia.

La distancia al poder manifiesta el grado de aceptación de la distribución inequitativa de poder por parte de los miembros menos poderosos de una sociedad, observándose el trato desigual entre las personas. Si existe un alto grado de distanciamiento al poder, las personas no requieren de una explicación o justificación frente a la situación de poder y se acepta un orden jerárquico en el cual todos tienen lugar. Si existe un bajo grado de distancia al poder, las personas tratan de igualar la distribución de poder y demandan explicaciones y justificaciones para las desigualdades en el poder.

El individualismo denota la preferencia por el cuidado de sí mismo y de las personas más cercanas (mayormente, la familia), esto genera una débil cohesión social, la dimensión opuesta es el colectivismo en donde los individuos esperan la inclusión y el cuidado del grupo, lo cual puede generar una alta cohesión social.

La aversión a la incertidumbre muestra el grado en el cual los miembros de un colectivo no están cómodos con la incertidumbre y con lo ambiguo, un alto grado de aversión a la incertidumbre puede expresarse por el mantenimiento de rígidos códigos de comportamiento y creencias inflexibles, no se toleran comportamientos e ideas heterodoxas. Un bajo grado de aversión a la incertidumbre se puede evidenciar con actitudes flexibles y tolerantes en la que la práctica es más relevante frente a rígidos principios establecidos.

\footnotetext{
Para más información consultar: http://geert-hofstede.com/ Dicho trabajo se basó en encuestas a empleados de IBM en todo el mundo acerca de los valores, las actitudes frente al trabajo y la situación laboral de la empresa, se realizaron a 100.000 empleados de la compañía en 63 países (Bhimani, 1999).
} 
La masculinidad exalta el logro, el heroísmo, la recompensa material por el éxito y la asertividad. La dimensión opuesta denominada feminidad exalta la modestia, la calidad de vida, el consenso, la preferencia por la cooperación y el cuidado del débil; probablemente el consenso puede ser alcanzado más fácilmente en esta dimensión opuesta.

La orientación a largo plazo se relaciona con la aceptación de la verdad de acuerdo al momento, situación y contexto, se muestra una mayor habilidad para adaptarse al cambio, se resalta la perseverancia para alcanzar las metas, además se puede relacionar con una fuerte propensión al ahorro y a la inversión. Lo contrario es la orientación al corto plazo, en la cual se quiere establecer una verdad absoluta, la normatividad del pensamiento y el gran respeto por las tradiciones es fundamental. El enfoque es hacia el logro de resultados rápidos en detrimento en ocasiones de los resultados futuros.

La indulgencia está relacionada con la permisividad relativamente libre hacia impulsos humanos relacionados con la diversión y con el goce de la vida. Lo opuesto se relaciona con la restricción, en donde se suprime la gratificación de necesidades y es regulada por estrictas normas de tipo social.

Además de las dimensiones de cultura nacional, en el Centro Hofstede ${ }^{4}$ se elaboró un modelo de cultura organizacional que consiste en seis dimensiones autónomas y dos dimensiones semiautónomas, dichas dimensiones están asociadas con la orientación hacia los medios versus la orientación a objetivos, disciplina de trabajo flexible y disciplina estricta, cultura local versus cultura profesional, sistemas cerrados y abiertos, orientación al empleado versus orientación al trabajo, grado de aceptación del estilo de liderazgo y grado de identificación con la organización. Estas dimensiones no han sido estudiadas de manera específica por la contabilidad, tal vez por lo reciente de su aparición en contraste con el modelo de culturas nacionales que ha permitido observar algunos elementos descriptivos de los sistemas contables de los países.

4 Ampliar información en: http://geert-hofstede.com/organisational-culture.html

\section{Investigación en contabilidad y cul- tura: descripción de los principales temas}

La línea de trabajo de contabilidad internacional y de manera específica de clasificación internacional de los sistemas contables ha sido importante para la caracterización y comprensión de las diferencias de sistemas y modelos contables a nivel mundial, esta línea que tuvo un enfoque hacia la contabilidad financiera, ha brindado elementos importantes para la contabilidad de gestión y para otras ramas de la contabilidad que se han interesado en comprender los sistemas contables a nivel mundial.

Harrison \& McKinnon (1999) son pioneros en incorporar la cultura dentro de los factores a tener en cuenta en el análisis de los cambios regulativos internacionales que afectan a los reportes financieros. Los valores y normas de los sistemas contables, así como el comportamiento de los grupos dentro y por fuera del sistema, son importantes para identificar la importancia de la cultura en dichos sistemas. El marco de trabajo de Hofstede (1980) se retoma en contabilidad para intentar explicar las diferencias de los sistemas contables a nivel internacional.

Gray (1988) afirma que la clasificación de los sistemas contables debe tener en cuenta además de los factores reconocidos como "importantes" (factores de orden económico o empresarial), los factores culturales, que no han tenido un reconocimiento o no han sido reconocidos en profundidad, aunque se incluyen como factores asociados al entorno. Gray (1988) intenta vincular las dimensiones de cultura nacional (Hofstede, 1980) para observar el desarrollo de los sistemas contables a un nivel de "subcultura" o de organización, para ello se enfoca en los valores, de manera específica en los valores relacionados con el trabajo, denominados "valores contables", que impactan eventualmente en los sistemas contables.

En primer lugar, se tiene la preferencia por el juicio profesional y la autorregulación profesional contrario a la conformidad de requerimientos estatutarios y el control que ejerce sobre el actuar profesional (pro- 
fesionalismo versus control estatutario). En segundo lugar, la preferencia por prácticas estándar y uniformes entre empresas y el uso de aquellas a través del tiempo, en oposición a la flexibilidad de acuerdo con los requerimientos de las organizaciones (uniformidad versus flexibilidad). En tercer lugar, la preferencia por prácticas conservadores en la medición para hacer frente a la incertidumbre de eventos futuros en oposición a aproximación que asumen un riesgo mayor (conservadurismo versus optimismo). Por último, se tiene la preferencia por la confidencialidad y la divulgación de la información sólo a los involucrados con la gestión de la organización, en oposición a una aproximación más transparente, pública y abierta (secreto versus transparencia).

Amat, Falguera, Puig \& Soldevilla (2003) afirman que la cultura es uno de los elementos que tienen influencia en los sistemas de contabilidad de gestión de los países, los sistemas de valores individuales en una sociedad influyen en las concepciones que se tienen en las organizaciones y repercute en sus prácticas. De manera particular, se identifica la preferencia por la regulación profesional versus la legislación oficial de la contabilidad, preferencia por la uniformidad versus flexibilidad de las técnicas contables, preferencia por el conservadurismo versus el optimismo en la valoración de las operaciones y preferencia por la confidencialidad de los datos versus la transferencia (Salgado \& Sánchez, 2012).

Jarne (1997) reconoce como un elemento que causa diferencias entre los sistemas contables de los países, la existencia de una subcultura contable y por tanto de valores culturales. El profesionalismo y la uniformidad tendrán que ver con el ejercicio de la autoridad en el sistema contable, así como con el nivel de exigencia en la aplicación normativa, el conservadurismo ejercerá su influencia con la adopción de unas prácticas de valoración más o menos prudentes, el secreto se verá plasmado en el nivel de información que es suministrado por la empresa al exterior (Salgado \& Sánchez, 2012).

Uno de los estudios más importantes en la revisión y crítica de la investigación cultural en contabilidad y control de gestión es el de Harrinson \& McKinnon (1999), que revisó los trabajos relacionados con el diseño de sistemas de control de gestión a nivel de comparación cultural ${ }^{5}$. Dicho trabajo, clarifica la intencionalidad de los investigadores y las dimensiones utilizadas para el análisis de los sistemas de control de gestión. Del anterior estudio se resaltan los siguientes trabajos: Lincoln, Hanada \& Olson (1981), estudiaron 522 compañías japonesas instaladas en Estados Unidos de América, utilizando el método de encuesta (mayormente utilizado en estos tipos de investigación), analizaron como características de los sistemas de control de gestión la diferenciación vertical y horizontal, utilizando la dependencia jerárquica, el rango y el paternalismo como categorías de análisis de nivel cultural. Birnbaum \& Wong (1985) estudiaron 93 bancos multinacionales instalados en Hong Kong, evaluaron como características la centralización, la diferenciación vertical y horizontal y la formalización de los sistemas de control de gestión (SCG), utilizaron las dimensiones de distancia al poder, aversión a la incertidumbre y jerarquía.

Frucot \& Shearon (1991) estudiaron la relación entre el locus de control, la participación en el presupuesto, desempeño y satisfacción laboral. Utilizaron las dimensiones de análisis de distancia al poder y aversión a la incertidumbre. Vance, McClaine, Boje \& Stage (1992) estudiaron organizaciones de Tailandia, Indonesia, malasia y EUA, en dicho estudio estudiaron la formalidad de las estructuras y controles, desarrollo individual versus desarrollo del grupo, involucramiento de los empleados en los procesos de evaluación, recompensas intrínsecas y extrínsecas, y frecuencia de feedback. Las dimensiones culturales utilizadas fueron la aversión a la incertidumbre, la distancia al poder y el individualismo. Harrison (1992) estudió 115 organizaciones de Singapur y 96 de Australia, analizó la relación entre la participación del presupuesto y la confianza en las medidas contables de rendimiento (énfasis en el presupuesto) en la evaluación de los gerentes; Harrison (1993) estudió estas mismas organizaciones pero analizó sólo la confianza en las medidas contables de rendimiento en un estilo de evaluación superior. En los dos estudios nombrados anteriormente, se utilizaron como dimensiones la distancia al poder y el individualismo. Ueno \& Wu (1993) estudiaron 205 empresas de EUA

La revisión completa se incluye en el Anexo. 
y 247 empresas en Japón, como características de los sistemas de control de gestión estudian la libertad presupuestal, la controlabilidad en la evaluación del desempeño, la duración del horizonte temporal en la evaluación del desempeño y en el proceso de planeación de planeación del presupuesto, y la estructura del proceso de planeación del presupuesto (reglas y procedimientos), como categorías asociadas a la cultura se utilizó el individualismo y la aversión a la incertidumbre.

Harrison, McKinnon, Panchapakesan \& Leung (1994) estudiaron 104 organizaciones de EUA, 140 de Australia, 65 de Singapur y 55 de Hong Kong, en dicho estudio se enfocaron en el diseño organizacional, la descentralización de los centros de responsabilidad, uso de técnicas cuantitativas en la planeación y el control, el horizonte de tiempo de la planeación y la toma de decisiones (grupal versus individual), y formalización. Las dimensiones estudiadas fueron la distancia al poder, el individualismo y la orientación a largo plazo. Chow, Kato \& Shields (1994), trabajaron 54 empresas en EUA y 39 japonesas, mediando la metodología de experimento estudiaron desde factores asociados a la organización, la incertidumbre del entorno, el peso jerárquico, la centralización, las interdependencias y las reglas formales. Desde el enfoque de la planeación estudiaron la planeación de arriba abajo y la dificultad de la estandarización. Desde la evaluación, se estudiaron los filtros de controlabilidad y la evaluación relativa, $y$ finalmente estudiaron las recompensas de manera específica las de carácter individual y los pagos preconcebidos. Las dimensiones analizadas fueron la distancia al poder, el individualismo, la aversión a la incertidumbre y la masculinidad.

Merchant, Chow \& Wu (1995) hicieron el estudio de 23 empresas de Taiwan y 54 empresas de EUA, mediante entrevistas en profundidad, estudiaron el tamaño de las recompensas y su dependencia con el desempeño, las recompensas basadas en el desempeño individual y en el grupal, los incentivos para el desempeño a largo plazo y las evaluaciones de desempeño objetivas versus las subjetivas. Las dimensiones utilizadas fueron la distancia al poder, el colectivismo, la orientación a largo plazo, la aversión a la incertidumbre y la masculinidad. Chow, Shields \&
Wu (1996), estudiaron la descentralización, la estructuración de actividades, el presupuesto participativo, los estándares, recompensas frente al desempeño, confianzas en las medidas contables de rendimiento y evaluación participativa del presupuesto. Las dimensiones evaluadas fueron la distancia al poder, el individualismo, la orientación a largo plazo, la aversión a la incertidumbre y la masculinidad.

Doupnik \& Salter (1995) estudiaron las prácticas contables de más de 50 países, estableciendo categorías de semejanzas entre países, las dimensiones utilizadas en el estudio fueron la masculinidad, la distancia al poder, la aversión a la incertidumbre y el individualismo, además incluyeron otras dimensiones tales como el sistema legal, la legislación tributaria, grado de desarrollo económico, niveles de inflación, niveles de educación y relaciones entre empresas con proveedores de capital. El orden de importancia de los anteriores factores fueron: el sistema legal, niveles de inflación, aversión a la incertidumbre, masculinidad, capitalización y grado de desarrollo económico. El estudio concluye que los factores del entorno y los factores culturales son necesarios para explicar el carácter individual de un sistema contable nacional. Otros trabajos se han enfocado en los efectos de la participación en la elaboración de presupuestos sobre la percepción de los sistemas de control de gestión y de desempeño. Tsui (2001) estudió gerentes en China y Occidentales radicados en Hong Kong, se confirmó que los gerentes chinos tienen una mayor distancia al poder en comparación con los occidentales, los gerentes occidentales tienen una orientación a corto plazo en comparación a los Chinos que tienen una orientación a largo plazo, finalmente, los gerentes chinos muestran un alto grado de colectivismo en comparación a los occidentales que muestran un alto grado de individualismo.

El trabajo de Merchant, Chow \& Wu (1995) plantea proposiciones con respecto a las diferencias que pueden existir en la remuneración, medición y evaluación del desempeño de los gerentes, los resultados muestran que personas con un alto nivel de individualismo prefieren evaluaciones del desempeño y recompensas basadas más en el desempeño individual que en el colectivo. Las personas con una alta distancia al poder, prefieren o aceptan la centraliza- 
ción en la toma de decisiones y una baja participación en procesos de toma de decisiones. Las personas con una alta aversión a la incertidumbre intentan tienden a confiar en reglamentos de conducta, la estructuración de actividades y la estandarización de procedimientos, para reducir al mínimo la incertidumbre. Las personas con un alto grado de masculinidad tienden a preferir recompensas basadas en el desempeño mientras que aquellas personas con alta feminidad prefieren que se asignen de acuerdo a la necesidad (Salgado \& Sánchez, 2012).

Algunos estudios abordan la importancia de los contextos, acontecimientos sociales y culturales, además del impacto que tienen sobre los sistemas contables, el trabajo realizado por Pourjalali \& Meek (1995) muestra resultados de una investigación en Irán después de la revolución Islámica que puso fin a 2.500 años de monarquía y la creación de la República Islámica de Irán en 1979, los cambios en las variables culturales se dieron por un bajo individualismo, debido a la fuerza colectiva y la unión dada en la revolución y la guerra. La influencia del islam se reflejó en un alto grado de masculinidad, la distancia al poder se reflejó en alto grado por la desigualdad generada por factores económicos y la guerra condujo a un orden más jerárquico. Debido a la inflación y a la crisis económica se presentaron altos grados de aversión a la incertidumbre. Los valores culturales de Gray (1988) fueron puestos a prueba y se determinó que de acuerdo a la evidencia analítica la teoría de los valores culturales es consistente, reflejando como tendencias de la contabilidad después de la revolución un bajo poder profesional y más control estatutario, una alta uniformidad, alto secreto y alto conservadurismo.

De acuerdo al trabajo anteriormente citado, Amat, Blake, Wraith \& Oliveras (1999), con el apoyo del trabajo de Hooper (1995) analizan el caso de España después de la muerte de Franco, e intentan explicar los cambios en la contabilidad durante la transición a un sistema democrático. Ante los cambios acaecidos se espera un movimiento hacia un individualismo más alto, una menor distancia al poder y un movimiento a una aversión a la incertidumbre más débil. Un conjunto de posibles influencias de los valores culturales sobre la contabilidad en España se puede predecir desde la contabilidad financiera al existir más profesionalismo que control estatal, más flexibilidad, menos conservadurismo y menos secreto. Desde la contabilidad de gestión se resalta también más orientación profesional que estatal, mayor diversidad de prácticas innovadoras y más estimaciones optimistas.

\section{Conclusiones y aspectos para discusión}

El concepto cultural se encuentra bajo un revestimiento de complejidad, en parte asegurada por las múltiples acepciones que se le han adjudicado, tanto así que ha adquirido una mala reputación en los círculos de la antropología, entre otras cuestiones por la vaguedad con que con demasiada frecuencia se ha invocado el concepto de cultura (Geertz, 2003). Es necesario entonces, estudiar los enfoques de la antropología cultural y analizar sus posibles aplicaciones a estudios de carácter particular, los enfoques y tendencias evolucionan y se actualizan al ritmo de las disciplinas que les dan origen.

El estudio de la cultura es fundamental ya que, sin las estructuras culturales, la conducta del hombre sería virtualmente ingobernable, sería un puro caos de actos sin finalidad y de estallidos de emociones, de suerte que su experiencia sería virtualmente amorfa. La cultura, la totalidad acumulada de esos esquemas o estructuras, no es sólo un ornamento de la existencia humana, sino que es una condición esencial de ella (Geertz, 2003). La cultura permite que el hombre supere los problemas específicos que enfrenta desde lo material o desde lo humano. La organización es el rasgo esencial de la cultura que vivimos y experimentamos, (Malinowski, 1948). En las organizaciones, se da la agrupación de seres humanos, bajo reglas técnicas y normas sociales que forman su conducta, dichas reglas o normas buscan una conducta ideal y es necesario en el análisis cultural distinguirlas de las actividades, es decir, se debe examinar el ideal con referencia a la realidad presente.

El tratamiento de la cultura alrededor de dimensiones culturales puede ser considerado como un reduccionismo (Schein, 2010), la investigación de cultura y contabilidad se ha hecho segmentada o "por partes" evitando que el tema se aborde en su integralidad, no todas las dimensiones se abordan en los estudios, 
ofreciendo la idea de que las partes separadas podrían dar elementos de comprensión del fenómeno estudiado, sin embargo, como fenómeno conectado y relacional, la cultura ofrece riqueza conceptual en la medida en que se aborda integralmente. Los estudios en contabilidad no han permitido establecer patrones sistemáticos para el estudio de las variables culturales con el fin de replicar y verificar hallazgos previos para establecer bases sólidas de conocimiento (Harrison \& McKinnon, 1999). Además, los marcos sobre los que se define la cultura y se estudia en contabilidad no han avanzado y en ocasiones ignoran aspectos importantes que sobre el tema han estudiado otras disciplinas sociales y humanas. La cultura como fenómeno multidimensional, puede mostrar desde diferentes enfoques el esfuerzo por comprender los problemas de un grupo social de manera más profunda. El marco de trabajo de Hofstede (1980) se ha utilizado para estudiar algunas variables culturales (sin trabajar de manera completa el marco en la mayoría de los casos) y su relación con algunos aspectos de la contabilidad, los estudios han buscado establecer inferencias estadísticas importantes, sin embargo, pasan por alto conceptos que son considerados fundamentales en la antropología cultural.

Algunas debilidades de los estudios en contabilidad y cultura pueden estar dados por: La escogencia de trabajar con algunas dimensiones sin tener en cuenta el marco integralmente, lo cual genera una posible omisión sobre los efectos de las otras dimensiones sobre los sistemas contables. Existe cierta superficialidad en asumir la uniformidad y unidimensionalidad para cada dimensión. La tendencia a darle importancia a las dimensiones en todos los contextos, sin tener en cuenta las particularidades de cada sociedad (Harrison \& McKinnon, 1999). Los investigadores escogen comparar contextos que para ellos resultan muy diferentes, sobre todo las culturas orientales y occidentales, la escogencia de estos contextos puede obedecer a la búsqueda de diferencias que son evidentes por los contextos en los cuales la cultura toma forma, una mayor precisión teórica podría aportar elementos que permitan observar evidencias entre culturas muy similares o entre subculturas.

El aumento de las operaciones internacionales de las organizaciones y la realidad de las compañías multinacionales, resalta la importancia de la cultura nacional, problemas como transferir los sistemas contables de un país a otro, denotan que el enfoque cultural podría aportar elementos que ayuden en la comprensión y diseño de los sistemas contables. La producción de estudios vinculados a la cultura y contabilidad son considerados como exploratorios y limitados (Chenhall, 2003), la explicación ofrecida se basa en que los estudios se realizan siguiendo diferentes combinaciones de las dimensiones culturales o bien, analizan diferentes características de los sistemas de contabilidad y control de gestión. Sin embargo, el campo de investigación de la cultura y organización tiene un gran potencial para el desarrollo teórico, la importancia de la cultura organizacional es tal, que es posible que pueda controlar a la cultura nacional, en este tema hay un espacio para la aplicación de paradigmas y métodos de la sociología y la antropología.

Las corrientes de investigación de tipo interpretativo pueden acercarse y complementar las visiones tradicionales relacionadas con las variables culturales y la contabilidad. El apoyo que puede brindar la antropología cultural es fundamental para establecer elementos de comprensión cada vez más profunda y con solidez teórica, elementos tales como las tradiciones culturales, el cuerpo de normas de grupos sociales, las costumbres, las creencias, los valores, las instituciones, las concepciones heredadas, los símbolos, los mecanismos de control y las visiones del mundo, pueden ser importantes para futuros estudios (Salgado \& Caballero, 2013b).

Un mejor conocimiento de las diferencias culturales en la contabilidad puede proporcionar una guía para el gobierno cuando redacta algunas normas y reglamentos pertinentes, pueden ayudar a tener una mejor comprensión de los profesionales de la contabilidad y su comportamiento, y proporcionar una visión para predecir la tendencia del desarrollo de contabilidad (Fang, 2007). La profesión contable debe contener y comprender los valores culturales y sociales del entorno, basado en el ejercicio de la contabilidad como una actividad socio-técnica, que implica recursos o técnicas tanto humanos como no humanos, así como la interacción entre los dos (Perera, 1994, citado en Hauriasi \& Davey, 2009). Estos 
valores contables, a su vez, explican y determinan la estructura y la práctica de la contabilidad, incluidos los principios básicos de la medición y la publicación que determinan las prácticas de información financiera (Salter, 1995).

Así pues, es posible resignificar el concepto de cultura que los estudios en contabilidad han establecido, una manera es recurrir a la antropología cultural estableciendo modelos teóricos que puedan complementar y validar los conceptos trabajados en la investigación de la contabilidad y la cultura. Los métodos de investigación también pueden enriquecerse y complementarse, buscando cada vez, ser más pertinentes con los fenómenos y estructuras estudiadas. El avance realizado por la investigación contable en el ámbito de la cultura es significativo e importante, se debe entonces avanzar hacia otros horizontes los cuales podremos llegar gracias a los aportes hechos por los investigadores. El avance de la investigación en este campo supone incluso de manera interesante generar un cambio cultural en la comunidad académica contable.

\section{Referencias}

Agbejule, A. (2011). Organizational culture and performance: the role of management accounting system. Journal of Applied Accounting Research, 12 (1), 74-89.

Amat, O., Blake, J., Wraith, P. \& Oliveras, E. (1999). Dimensions of national culture and the accounting environment - The Spanish case. Department of Economics and Business, Universitat Pompeu Fabra.

Amat, O., Falguera, J., Puig, X. \& Soldevilla, P. (2003). Aspectos que influyen en las diferencias internacionales en materia de contabilidad de gestión. Revista iberoamericana de contabilidad de gestión, 1(1).

Aktouf, O. (2002). El simbolismo y "la cultura organizacional". Revista Ad-Minister, 1.

Batool, S. (2011). Analyze the Factors that have an Influence on the Management Control System. Research Journal of Finance and Accounting, 2 (3), 48-56.

Berry, A., Broadbent, J. \& Otley, D. (2005). Management Control: Theories, Issues and Performance. New York, Palgrave.

Bhimani, A. (1999). Mapping methodological frontiers in crossnational management control research. Accounting, Organizations and Society, 413 - 440.

Birnbaum, P. H., \& Wong, G. Y. Y. (1985). Organizational structure of multinational banks in Hong Kong from a culture-free perspective. Administrative Science Quarterly, 30, 262-277.

Busco, C. \& Scapens, R. (2011). Management accounting systems and organisational culture. Qualitative Research in Accounting and Management, 8(4), 320-357.
Chenhall, R. (2003). Management control systems design within its organizational context: findings from contingency-based research and directions for the future. Accounting, Organizations and Society, 28, 127-168.

Chow, C. Shields, M. \& Chan, Y. (1991). The efects of management controls and national culture on manufacturing performance: an experimental investigation. Accounting, Organizations and Society, 16, 209-226.

Chow, C. W., Kato, Y. \& Shields, M. D. (1994). National culture and the preference for management controls: an exploratory study of the firm-labor market interface. Accounting, Organizations and Society, 19, 381-400.

Chow, C. W., Shields, M. D. \& Wu, A. (1996). The importance of national culture in the design of and preference for management controls for multinational operations. Paper presented at the Accounting, Organizations and Society Comparative Management Accounting Conference, University of Siena, Italy.

Doupnik, T.S. \& Salter, S.B. (1995). External environment, Culture and Accounting Practice: A Preliminary Test of A General Model of International Accounting Development. International Journal of Accounting, 189-207.

Fang, Y. (2007). On accounting and culture/Comptabilité et culture. Canadian Social Science, 3 (5), 121-124.

Frucot, V. \& Shearon, W. T. (1991). Budgetary participation, locus of control, and Mexican managerial performance and job satisfaction. The Accounting Review, 66, 80-99.

Geertz, C. (2003) La interpretación de las culturas. Gedisa, México.

Giménez, G. (2000). Territorio, cultura e identidades. Cultura y región. CES, Universidad Nacional de Colombia, Ministerio de Cultura. Colombia.

Giménez, G. (2007). Un recorrido a través del concepto de cultura. Estudios sobre la cultura y las identidades sociales. Conaculta - Iteso. México.

Gray, S. J. (1988). Towards a Theory of Cultural Influence on the Development of Accounting Systems Internationally. ABACUS, 24(1), $1-15$.

Harris, M. (2009). Antropología cultural. Decima reimpresión. Alianza editorial, Madrid.

Harrison, G. L. (1992). The cross-cultural generalizability of the relation between participation, budget emphasis and job related attitudes. Accounting, Organizations and Society, 17, 1-15.

Harrison, G. L. (1993). Reliance on accounting performance measures in superior evaluative style, the influence of national culture and personality. Accounting, Organizations and Society, 18, 319-339.

Harrison, G. L. \& McKinnon, J. L. (1999). Cross-cultural research in management control systems design: a review of the current state. Accounting, Organizations and Society, 483 - 506.

Harrison, G. L., McKinnon, J. L., Panchapakesan, S. \& Leung, M. (1994). The influence of culture on organizational design and planning and control in Australia and the United States compared with Singapore and Hong Kong. Journal of International Financial Management and Accounting, 5, 242-261.

Hauriasi, A. \& Davey, H. (2009). Accounting and culture: The case of Solomon Islands. Pacific Accounting Review, 21, 228 -259.

Heidhues, E. \& Patel, C. (2011). A critique of Gray's framework on accounting values using Germany as a case study. Critical Perspectives on Accounting, 22(3), 273-287.

Herath, K. (2007). A Framework for Management Control Research. Journal of management development. 
Hofstede, G. (1980). Culture's consequences: International differences in work-related values. Beverly Hills, California. Sage.

Hofstede, G. (1984). The cultural relativity of the quality of life concept. Academy of Management Review, 27, $389-398$.

Hofstede, G. (2011) Dimensionalizing Cultures: The Hofstede Model in Context. Online Readings in Psychology and Culture, 2(1), http://dx.doi.org/10.9707/2307-0919.1014.

Hooper, J. (1995). The New Spaniards. Penguin Books. London.

Jarne, J.I. (1997). Clasificación y evolución internacional de los sistemas contables. Asociación Española de Contabilidad y Administración de Empresas. Madrid.

Kottak, C. (2006). Antropología Cultural. Traducción Lisón J., Mc Graw Hill, Madrid.

Lincoln, J. R., Hanada, M. \& Olson, J. (1981). Cultural orientations and individual reactions to organizations: a study of employees of Japanese-owned firms. Administrative Science Quarterly, 26, 93-115.

Malinowski, B. (1948). Una teoría científica de la cultura y otros ensayos. Editorial Sudamericana, Buenos Aires.

Merchant, K. \& Otley, D. (2007). A review of the literature on control and accountability. Handbook of Management Accounting Research. London. Elsevier.

Merchant, K. A., Chow, C. W. \& Wu, A. (1995). Measurement, evaluation and reward of profit center managers: a cross-cultural field study. Accounting, Organizations and Society, 20, 619-638.

Ouchi, W. (1979). A conceptual framework for the design of organizational control systems. Management Science.

Pourjalali, H. \& Meek, G. (1995). Accounting and culture: the case of Iran. Research in Accounting in Emerging Economies, 3, 3-17.

Salgado, C. \& Sánchez, D. (2012). El concepto cultural en la Contabilidad de Gestión: una mirada y una crítica. En: VIII congreso iberoamericano de administración empresarial y contabilidad y VI congreso iberoamericano de contabilidad de gestión Pontificia Universidad Católica del Perú. Lima (Perú).

Salgado, J. \& Caballero, D (2013). Influencia de las variables culturales sobre los sistemas de contabilidad de gestión: aproximación a un modelo conceptual. En: VII Congreso Iberoamericano de Contabilidad de Gestión y IX Congreso Iberoamericano de Administración Empresarial y Contabilidad. Universidad de Valencia. Valencia (España).

Salgado, J. \& Caballero, D (2013). Estudio de las variables culturales desde la contabilidad: una revisión a los métodos de investigación. En: Encuentro Internacional de Investigadores en Administración 2013. Universidad Externado, Universidad del Valle, Universidad del Magdalena. Santa Marta (Colombia).

Salgado, J. (2015). Lo lóbrego del control de gestión. En: I Jornada Internacional de Estudios Disciplinares en Contabilidad. Universidad Militar Nueva Granada. Bogotá (Colombia)

Salter, S. \& Niswander, F. (1995). Cultural influence on the development of accounting systems internationally: a test of Gray's (1988) theory, Journal of International Business Studies, 26, 379-397.

Schein, E. H. (2010). Organizational culture and leadership. San Francisco, CA.: Jossey Bass.

Tsui, J. S. (2001). The impact of culture on the relatioship between budgetary participation, management accounting systems and managerial performance: An analysis of Chinese and Western managers. International Journal of Accounting, 36, 125 - 146.

Ueno, S. \& Wu, F. H. (1993). The comparative influence of culture on budget control practices in the United States and Japan. International Journal of Accounting, 28, 17-39.

Vance, C. M., McClaine, S. R., Boje, D. M. \& Stage, D. (1992). An examination of the transferability of traditional performance appraisal principles across cultural boundaries. Management International Review, 32, 313-326. 
ANEXO. Estudios culturales comparativos de diseño de sistemas de control de gestión (1980-1996)

\begin{tabular}{|c|c|c|c|c|c|c|c|c|c|c|}
\hline 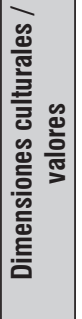 & 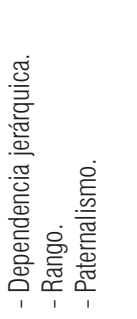 & 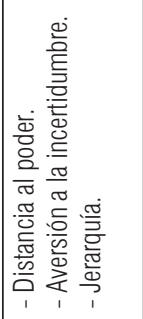 & 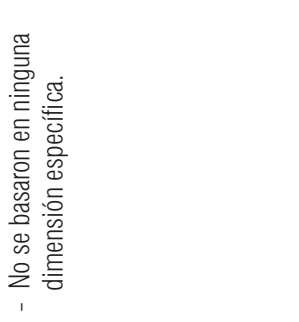 & 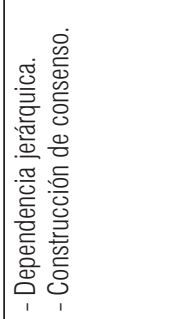 & 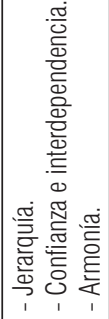 & 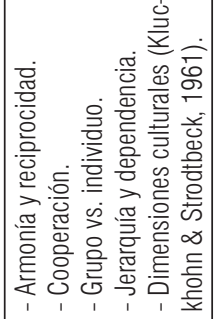 & 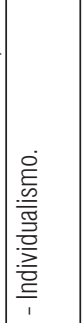 & 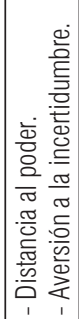 & 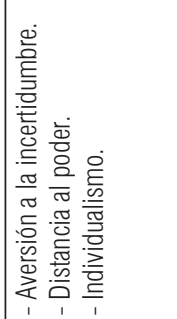 & 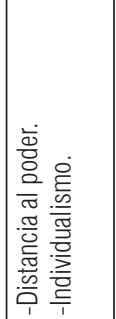 \\
\hline 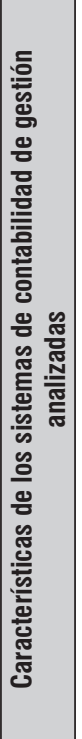 & 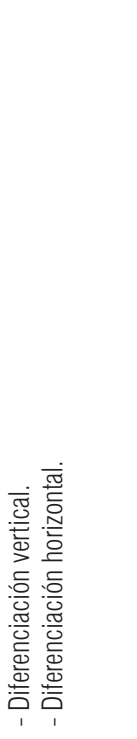 & 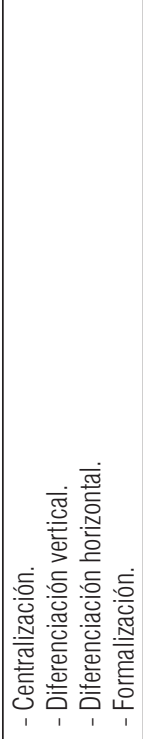 & 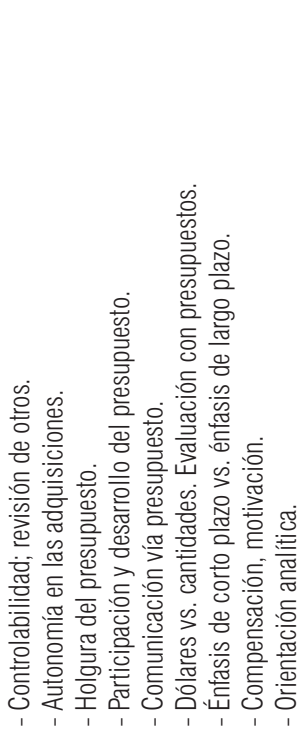 & 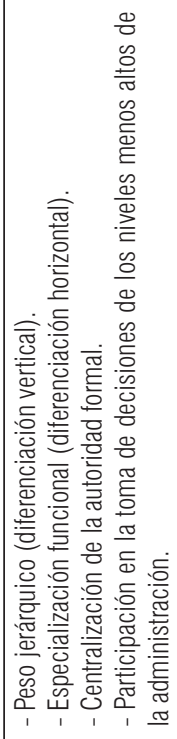 & 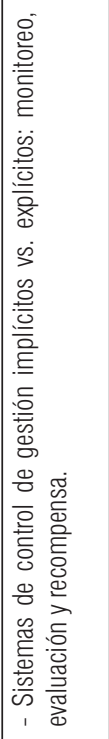 & 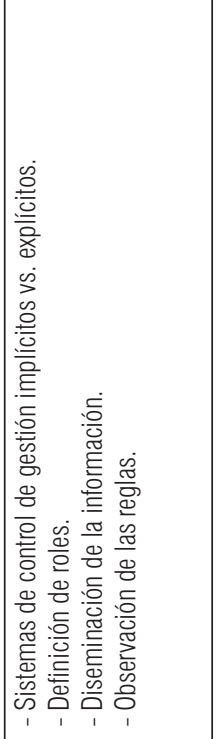 & 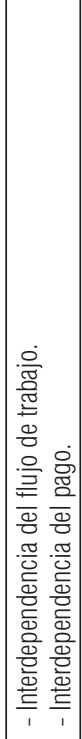 & 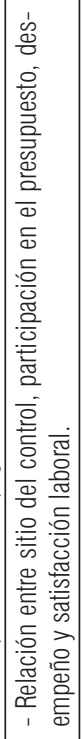 & 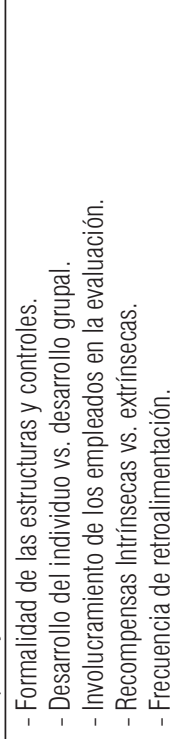 & 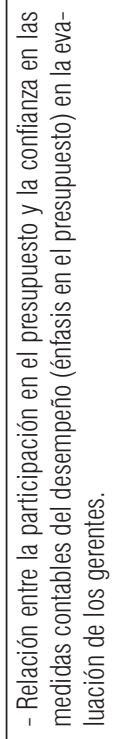 \\
\hline 음 & 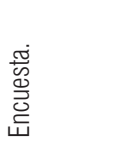 & 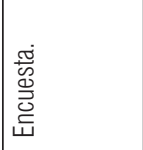 & $\begin{array}{l}\text { 密 } \\
\text { 产 } \\
\text { 岕 }\end{array}$ & 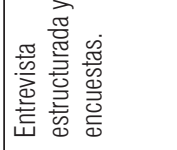 & 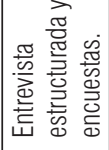 & 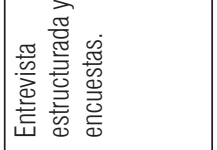 & 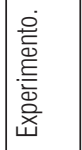 & 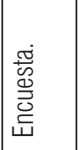 & 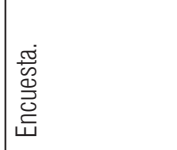 & 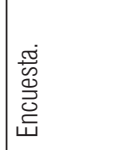 \\
\hline 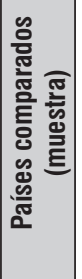 & 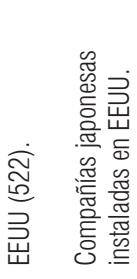 & 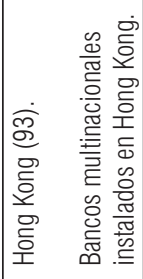 & 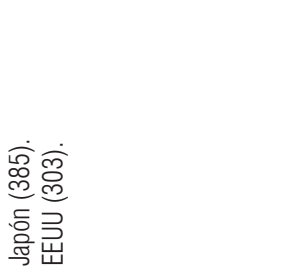 & 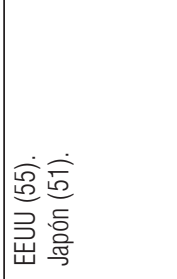 & 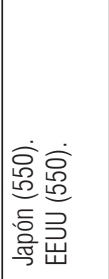 & 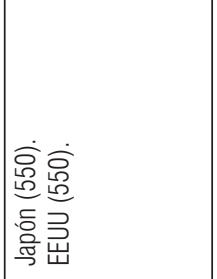 & 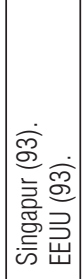 & 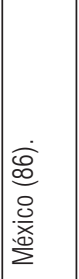 & 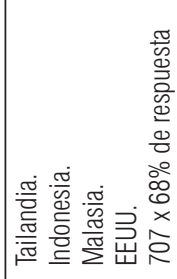 & 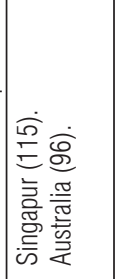 \\
\hline $\begin{array}{l}\text { 을 } \\
\text { 离 }\end{array}$ & 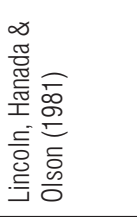 & 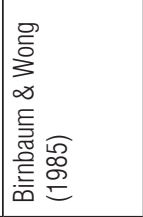 & 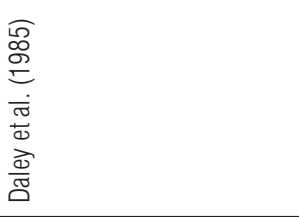 & 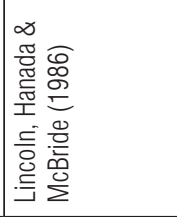 & 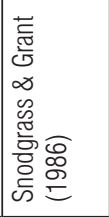 & 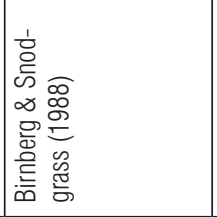 & 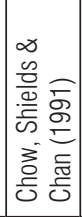 & 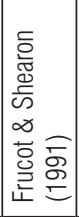 & 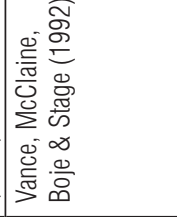 & 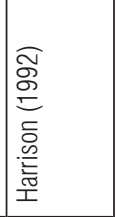 \\
\hline
\end{tabular}




\begin{tabular}{|c|c|c|c|c|c|c|c|c|c|c|}
\hline 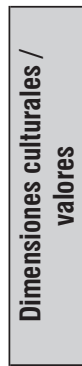 & 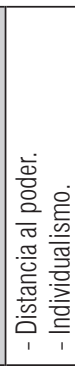 & 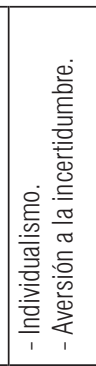 & 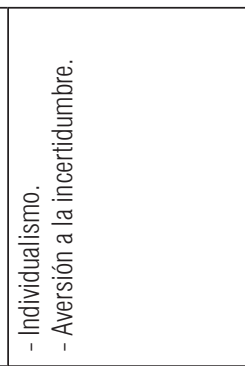 & 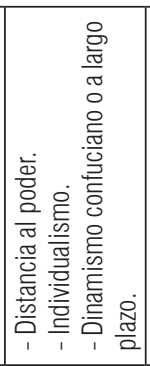 & 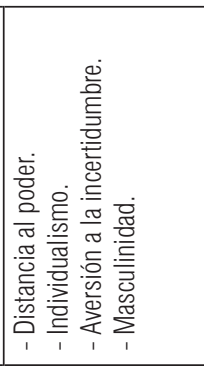 & 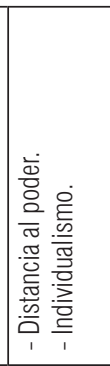 & 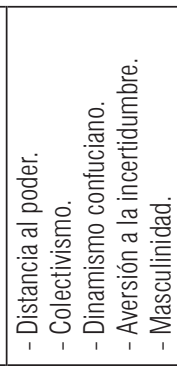 & 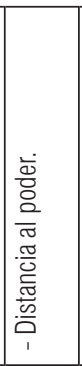 & 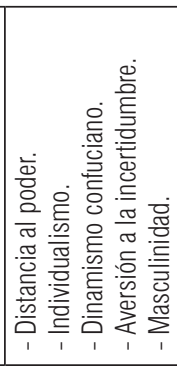 & 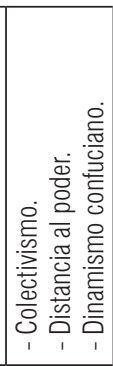 \\
\hline 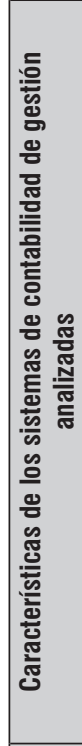 & 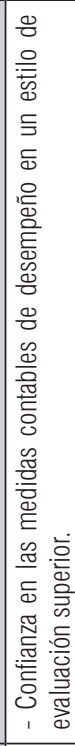 & 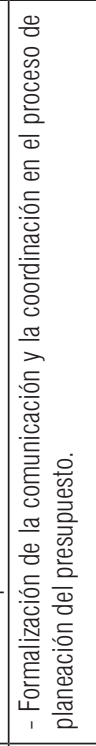 & 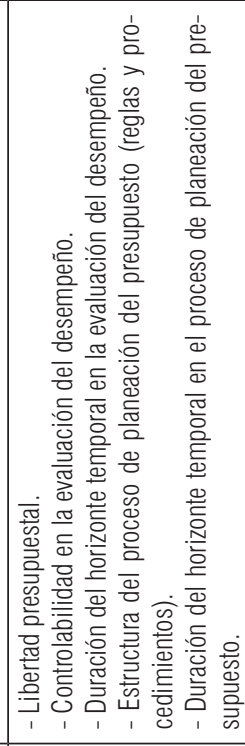 & 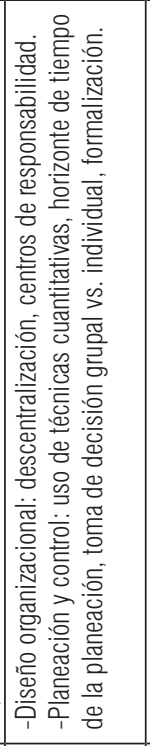 & 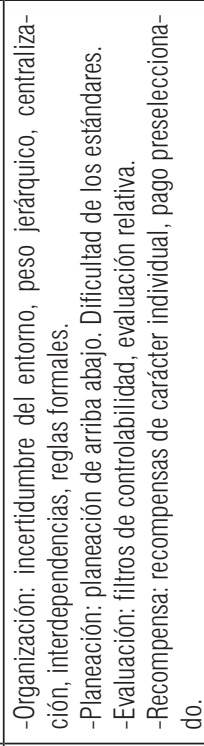 & 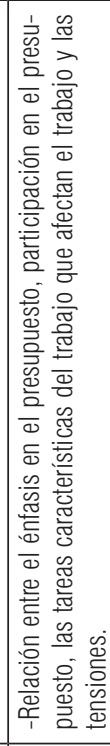 & 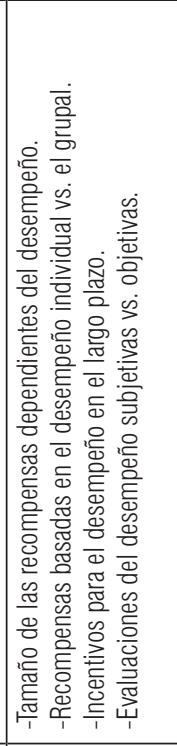 & 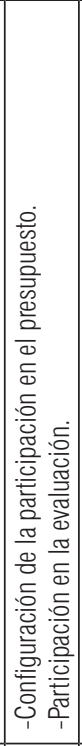 & 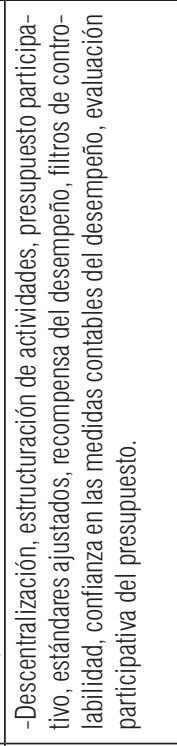 & 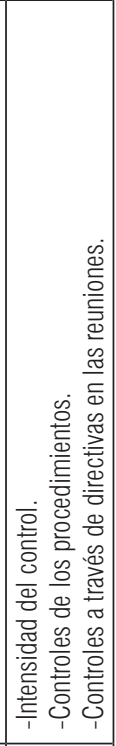 \\
\hline $\begin{array}{l}\text { 음 } \\
\text { 일 }\end{array}$ & 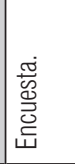 & 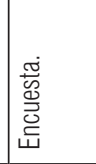 & 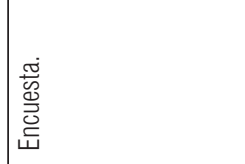 & 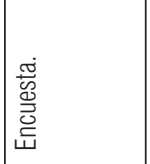 & 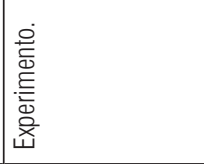 & 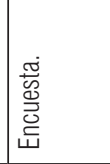 & 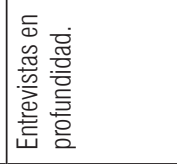 & 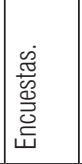 & 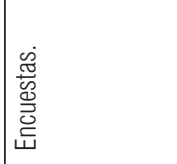 & 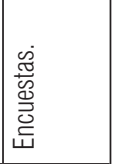 \\
\hline 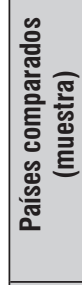 & 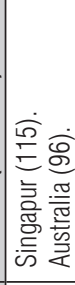 & 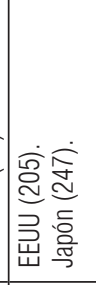 & 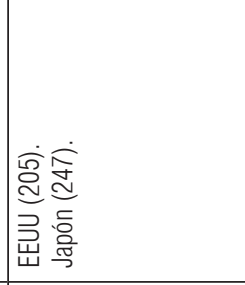 & 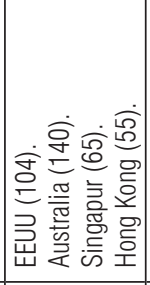 & 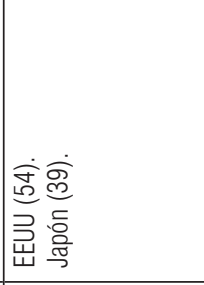 & 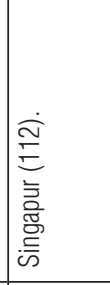 & 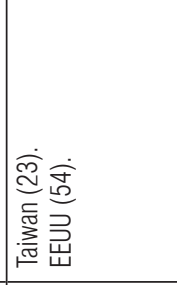 & 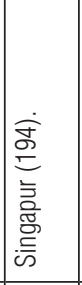 & 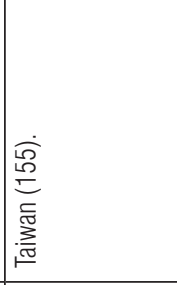 & 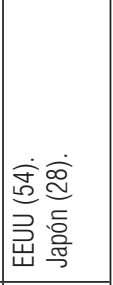 \\
\hline $\begin{array}{l}\text { 을 } \\
\text { 离 }\end{array}$ & 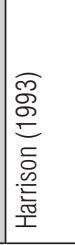 & 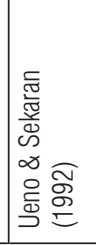 & 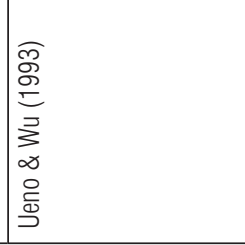 & 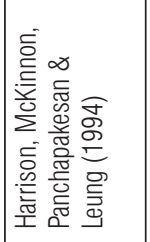 & 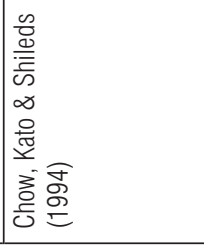 & 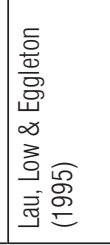 & 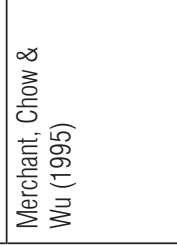 & 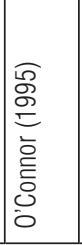 & 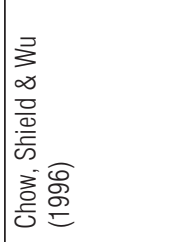 & 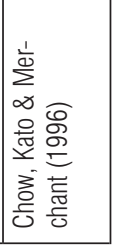 \\
\hline
\end{tabular}

